\title{
Peculiarities of quasicrystalline films formation processes on crystal surface under irradiation with proton-ion flows
}

\author{
Gennady Melnikov ${ }^{1, *}$, Nikolay Ignatenko ${ }^{1}$, Lyudmila Petrova ${ }^{1}$, Vadim Suchilkin ${ }^{1}$ and \\ Andrey Gromkov ${ }^{1}$ \\ ${ }^{1}$ Southwest State University, 305040 Kursk, Russia
}

\begin{abstract}
Quasicrystalline film with densest package of atoms can be formed on a crystal surface under irradiating crystal surface with protonion flows at a certain ratio of atoms diameters of irradiated crystal and the ions of irradiating flow. Atom-free area of $1 \AA$ order is formed. These are traps for protons from the irradiation stream, thus a quantum dot appears. Atomic package of quasicrystalline film is a package of equilateral Penrose rhombs and there are centers of atoms mass at the vertices. A mathematical relation is obtained that allows predicting radii of irradiation flux ions to form quasicrystalline film and select atomic composition of the obtained film with predetermined properties. Nanostructuring of materials is an entire family of physicochemical processes associated with proton transfer and their localization in crystal lattice and these include ion exchange, diffusion, and ion implantation. Ion and proton exchange can be considered an established universal method of surface modification technology [2426]. The case of implantation of protons into the structure of cluster systems formed on the surface of crystals is described in this paper. In this case, a quantum dot is formed in the cluster structure, which is a potential hole with quantized proton motion, wherein the radiation of quantum dot is in IR area of electromagnetic spectrum.
\end{abstract}

\section{Introduction}

Quasicrystals is a large class of ordered substances that occupy an intermediate position between classical crystals and amorphous substances. The first quasicrystal was discovered by Schechtman in 1984. It was discovered by using rapid cooling of the melt [1]. More than a hundred systems based on aluminum, gallium, copper, cadmium, nickel, titanium, tantalum and other elements have been discovered. Quasicrystals [1, 2] are formed on its basis.

Mathematical model of an ideal quasicrystal was developed in two independent theories, which later turned out to be equivalent. In [3, 4], a model of a quasicrystal is built, based on two elementary cells with an irrational ratio of their quantities. Certain rules of

\footnotetext{
* Corresponding author: melnikovga@mail.ru
} 
construction, based on Fibonacci numerical sequence, led to a structure in which periodicity in atoms arrangement is absent, but there are several properties typical for the crystal.

A common model of quasicrystalline object's structure is a two-fragmental model based on a quasi-periodic coating of a straight line, plane, or space with two elemental structural units. This model leads to a Fibonacci sequence of short $S$ and long $L$ segments with $S=1$ and $\mathrm{L}=\Phi=1.6180339 \ldots$. In two-dimensional case, two-fragmental model is a Penrose tiling [4]. It consists of two types of rhombs with sharp angles $\pi / 5$ and $2 \pi / 5$. In threedimensional case, quasicrystal structure is formed by rhombohedra of two types and is a generalization of Penrose tiling. It is called Amman-Mackay structure [6, 7].

Icosahedral quasicrystal model structure was obtained in Kalugin, Kitaev, Levitov model [5] which is based on «cubic» crystal in 6-dimensional space. So-called «pipe» is cut from this crystal. This «pipe» is a layer of atoms with an order of interatomic magnitude, enclosed between three-dimensional hyperplanes. Then it is projected onto physical space.

This construction allows us to obtain a continual theory of a quasicrystal, describe dislocations and find out principles of low-frequency collective modes, in addition to ordinary phonons, arising from three degrees of atoms free motion along the pipe.

Cluster approach [8-10] states that quasicrystals are aggregates of clusters. Their structure cannot be represented as a package of identical elementary cells. They are a unique method of packing mutually overlapping clusters, which allows the implementation of the most stable, energetically preferred local atomic configurations. In Penrose partition, two types of clusters of three rhombuses can be distinguished, resembling cube projections in shape. This is either a cluster of two wide and one narrow rhombus, or an elongated cluster of one wide and two narrow rhombuses. Any rhombus is always a part of either one or two mutually overlapping clusters of these types [8-10].

One-dimensional model of a quasicrystal and numerical Fibonacci sequences associated with Golden Section and is used to build a new type of optical elements: aperiodic diffraction gratings and multilayer structures [8-12].

Two-fragmental model based on quasi-periodic coverage of space by two elementary structural units is a model basis of cluster systems structure adopted by the authors of this paper.

According to Penrose tiling model, elementary units of the structure are thin and thick rhombuses with equal $a$ sides, at vertices of which there are centers of atoms mass. A thin rhombus has an acute angle $\pi / 5=36^{\circ}$ and a thick rhombus with an acute angle $2 \pi / 5=72^{\circ}$. Atoms at peaks of thin rhombus form two dimers (Figure 1) and atoms form two more dimers at the peaks of a thick rhombus.

The first dimer is formed by opposing atoms of a thin rhombus, wherein the centers of atoms mass are located at a distance of a small rhombus diagonal:

$$
R_{\mathrm{dim}}^{\prime}=2 a \sin \frac{\pi}{10}=a(\Phi-1)=\frac{a}{\Phi}=0.618 \ldots \cdot a,
$$

the second dimer is formed by atoms at a distance of a large diagonal of a thin rhombus:

$$
R_{\mathrm{dim}}^{\prime \prime}=2 a \cos \frac{\pi}{10}=a \sqrt{\Phi+2}=1.902 \ldots \cdot a,
$$

where

$$
R_{\mathrm{dim}}^{\prime \prime}=R_{\mathrm{dim}}^{\prime} \operatorname{ctg} \frac{\pi}{10}=R_{\mathrm{dim}}^{\prime} \frac{\sqrt{\Phi+2}}{\Phi-1}=R_{\mathrm{dim}}^{\prime} \Phi \sqrt{\Phi+2}=3.078 \ldots \cdot R_{\mathrm{dim}}^{\prime},
$$


where $\Phi=1.6180339 \ldots$ - «golden» ratio.
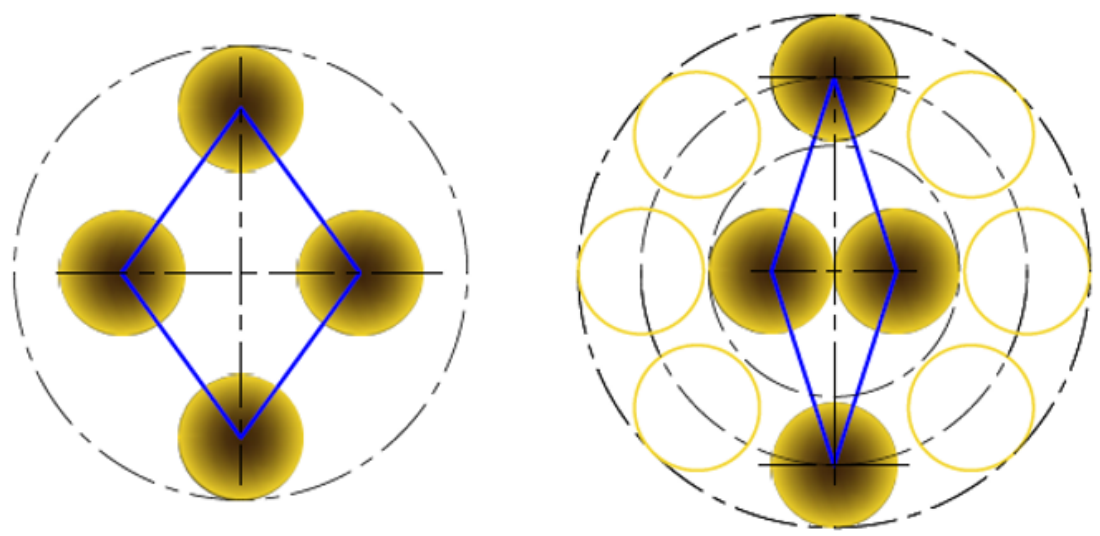

Fig. 1. The formation of atoms dimers in the structure of clusters according to «golden» rhombuses scheme.

Atoms at the vertices of a thick rhombus, by analogy with a thin rhombus, form two more dimers with distances between atoms $R_{\mathrm{dim}}^{\prime \prime \prime}$ and $R_{\mathrm{dim}}^{I V}$ respectively

$$
\begin{gathered}
R_{\mathrm{dim}}^{\prime \prime \prime}=2 a \sin \frac{\pi}{5}=a \sqrt{3-\Phi}=1.175 \ldots \cdot a, \\
R_{\mathrm{dim}}^{I V}=2 a \cos \frac{\pi}{5}=a \Phi=1.618 \ldots \cdot a, \\
R_{\mathrm{dim}}^{I V}=R_{\mathrm{dim}}^{\prime \prime \prime} \operatorname{ctg} \frac{\pi}{5}=R_{\mathrm{dim}}^{\prime \prime \prime} \frac{\Phi}{\sqrt{3-\Phi}}=1.377 \ldots \cdot R_{\mathrm{dim}}^{\prime \prime \prime} .
\end{gathered}
$$

Each of four dimers becomes nucleus of the emerging clusters. So, in such a model it is necessary to distinguish four types of clusters, each of them forms a diffraction pattern in $\mathrm{X}$-ray-electron structural studies. The overall X-ray pattern is the result of the overlay of four types of reflexes displaying the features of four cluster's types in the structure of quasicrystalline system.

Radii of coordination spheres are represented by the ratio for the system of successive coordination spheres of spherical particles [11-14]

$$
R_{n}=R_{d i n} \sqrt{p F_{n}}
$$

where $R_{\text {dim }}$ - distance between particles in each of the four dimers (1)-(6), $F_{n}$ - Fibonacci numbers, $p=1,2,3 \ldots-$ the positive integers.

Table 1 shows results of radii calculations of coordination spheres in units of rhombus side $\alpha$ for four types of clusters in structure of quasicrystalline system according to formula (7) where $p=1$.

Rhombus $a$ side can be estimated using formula (1), in which it is necessary to state the radius of the first coordination sphere $\left(R_{\mathrm{dim}}^{\prime}\right)$, which is determined by the particle diameter of the substance and features of interaction potential [11-14] 


$$
R_{1}=R_{\mathrm{dim}}^{\prime \prime \prime}\left(\frac{n}{m}\right)^{\frac{1}{n-m}} \sigma_{0}
$$

where $R_{1}$ is the radius of the first coordination sphere, $\sigma_{0}$ is the diameter of the sphere modeling the atom, $n>m$ - are integers for $\mathrm{t}$ Mi potential.

Table 1. Radii of successive coordination spheres in $a$ units in the structure of a quasicrystalline system.

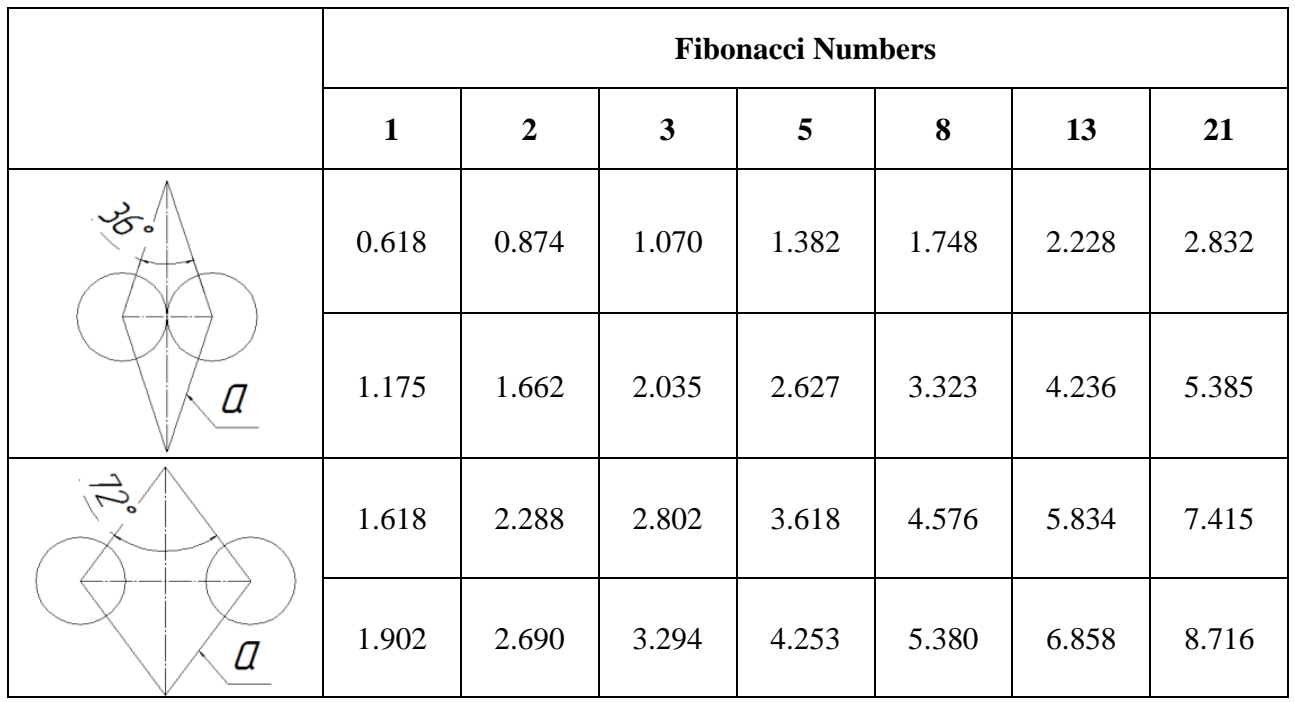

Considering made assumptions, the side of the rhombus will be determined by the following formula

$$
a=\Phi\left(\frac{n}{m}\right)^{\frac{1}{n-m}} \sigma_{0}
$$

The radius of the atom will be considered half the radius of the first coordination sphere of a radial distribution function for a pure substance with a certain variety of atoms, for atoms radii of two classes we write the following:

$$
r_{1}=\frac{R_{1}^{\prime}}{2}=\frac{1}{2}\left(\frac{n_{1}}{6}\right)^{\frac{1}{n_{1}-6}} \sigma_{01}, \quad r_{2}=\frac{R_{1}^{\prime \prime}}{2}=\frac{1}{2}\left(\frac{n_{2}}{6}\right)^{\frac{1}{n_{2}-6}} \sigma_{02},
$$

where $m=6$ is accepted. That corresponds to the dispersion forces of attraction (in the case of Mi power potential, atoms radii depend on degree indicators in repelling and attracting parts of the potential).

Based on Table 1, we obtain absolute values of radii coordination spheres at $a=2.804 \AA$ of rhombus side by arranging coordination spheres radii in order of monotonic increase of their values.

Short-range order (in five coordination areas) in densely packed carbon systems can be modeled with quasicrystalline models.

Quasicrystalline models of cluster systems structure in the structure of carbon films make it possible to predict the position of coordination areas consistent with the results of 
X-ray structural measurements. The consistent arrangement of coordination areas is explained by the features of the Fibonacci series and «golden» ratio.

The study of formation and stability problem of thin films in the system of double solid solutions revealed several conditions when selecting film components. First, it is necessary to implement the principle of densest packing of atoms or molecules in the structure of the resulting film, which corresponds to minimum potential energy of the original cluster during thin film formation [21-23].

We obtain the condition of densest packing for particles of two grades under a certain ratio between radii atoms of the irradiated surface and atoms (ions) radius of the irradiating flux $r_{2}>r_{1}$, considering the properties of a «golden» rhombus. According to Figure 2 radii are associated with the trigonometric functions of the «golden» angles:

$$
r_{2}=r_{1}\left(\frac{2}{2 \sin \alpha}-1\right) \text {. }
$$

Considering ratio (10), formula (11) will be the following:

$$
\frac{r_{2}}{r_{1}}=\frac{n_{2}^{\frac{1}{n_{2}-6}}}{n_{1}^{\frac{1}{n_{1}-6}}} \cdot 6^{\frac{n_{2}-n_{1}}{\left(n_{1}-6\right)\left(n_{2}-6\right)}}\left(\frac{2}{2 \sin \alpha}-1\right) \cdot \frac{\sigma_{02}}{\sigma_{01}},
$$

where $n_{1}, n_{2}$ are the degree indices in the repulsive part of Mi paired interaction potential for two classes of atoms, $\sigma_{01}, \sigma_{02}$ are the diameters of solid spheres modeling these atoms.
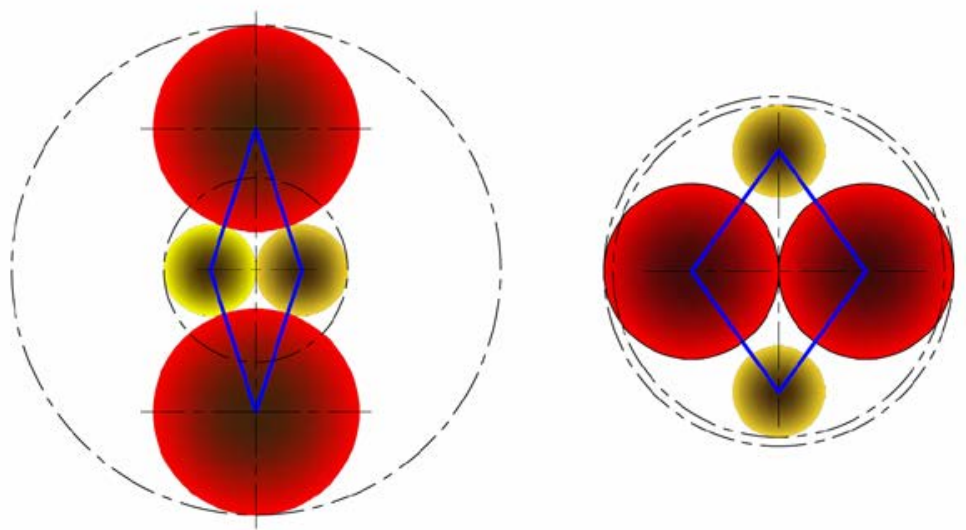

Fig. 2. Tight packing of two grades particles in the model of «golden» rhombuses.

Table 2 shows values of «golden» angles in thin and thick rhombuses and the sinuses of these angles expressed through the «golden» ratio, which show that a certain ratio between the radii of the constituent particles as a function of a "golden» ratio is necessary to implement the principle of dense particles packing.

Here are some examples.

The radius of fluorine atom is $r_{1}(\mathrm{~F})=0.73 \AA$, therefore, according to Table 2 and Figure 2, atoms with $r_{2}=1.63 \AA ; 0.51 \AA ; 0.172 \AA$ radii are the most preferred atoms for forming a dense package of atoms in a thin film. 
Table 2. Trigonometric functions of «golden» angles.

\begin{tabular}{|c|c|c|c|c|c|}
\hline \multirow{2}{*}{ Function } & \multicolumn{5}{|c|}{ Parameter } \\
\cline { 2 - 6 } & $\pi / 10=18^{\circ}$ & $\pi / 5=36^{\circ}$ & $3 \pi / 10=54^{\circ}$ & $2 \pi / 10=72^{\circ}$ & $3 \pi / 5=108^{\circ}$ \\
\hline $2 \sin \alpha$ & $\Phi-1$ & $\sqrt{3-\Phi}$ & $\Phi$ & $\sqrt{\Phi+2}$ & $\sqrt{\Phi+2}$ \\
\hline $2 \cos \alpha$ & $\sqrt{\Phi+2}$ & $\Phi$ & $\sqrt{3-\Phi}$ & $\Phi-1$ & $1-\Phi$ \\
\hline$r_{1} / r_{2}$ & 2.236 & 0.7013 & 0.2361 & 0.05146 & 0.05146 \\
\hline
\end{tabular}

The radius of the magnesium atom $r_{2}(\mathrm{Mg})=1.60 \AA$ can form a «golden» rhombus with an angle $\pi / 5=36^{\circ}$ in combination with fluorine atom. Such compound is magnesium fluoride $\left(\mathrm{MgF}_{2}\right)$, which is, under normal conditions, colorless diamagnetic tetrahedral crystals with $P 4 / m n n(a=4.625 \AA ; c=3.052 \AA ; Z=2)$ spatial group.

The second value for the ions of $r_{2}=0.51 \AA$ irradiation stream is close to the radius of hydrogen atom to form a hydrogen fluoride (HF) compound - a colorless gas (under standard conditions) with a sharp odor. at room temperature it exists mainly in the form of a dimer $\mathrm{H}_{2} \mathrm{~F}_{2}$ at room temperature and this is a colorless movable fluid if the temperature is below $19.9^{\circ} \mathrm{C}$.

The radius of silicon atom (ion) is $r_{1}(\mathrm{Si})=1.32 \AA$. Atoms with radii $r_{2}=0.926 \AA ; 0.51 \AA ; 0.172 \AA$ are preferable to form a dense package of atoms in siliconbased thin film. The radius of $r_{2}(\mathrm{Br})=0.94 \AA$ bromine atom in combination with silicon atom can form a "golden" rhombus and silicon bromide compound (II) - a binary inorganic compound of silicon and bromine with $\mathrm{SiBr}_{2}$ formula. A substance with the color from yellow to brown polymerizes to $\left(\mathrm{SiBr}_{2}\right)_{\mathrm{n}}$ with a molecular weight of $3000-3600$. At $60^{\circ} \mathrm{C}$ it softens, at $100-110^{\circ} \mathrm{C}$ it is drawn into threads, at $160-180^{\circ} \mathrm{C}$ it becomes viscous, like a lubricating oil.

Zinc oxide is technologically important inorganic compound with $\mathrm{ZnO}$ formula. The radius of oxygen atom is $r_{1}(\mathrm{O})=0.617 \AA$, the radius of zinc atom is $r_{2}(\mathrm{Zn})=2.236 \cdot r_{1}=1.342 \AA$. This corresponds to the formation of a «golden» rhombus. In a stable form, zinc oxide has a hexagonal crystal structure with lattice parameters $a=3.2458 \AA$ and $c=5.2006 \AA(c / a=1.6022)$. Materials based on $\mathrm{ZnO}$ have electrical conductivity of p-type. Zinc oxide thin films generally doped with Group III elements such as $\mathrm{Ga}$, In or Al. They are electrically conductive.

According to formula (11) $r_{2}=2.236 \cdot r_{1}$ irradiation flux corresponds to of cyclic hydrocarbon molecules - arenes: benzene, toluene, xylene, etc.). This is suitable for ions atoms radii. Molecules of these hydrocarbons and their halogen substituted form a class of organometallic compounds (MOS) in combination with metal atoms. The interaction of cyclic hydrocarbons with metal atoms can form stable cluster systems and form a logical class of substances.

Atoms with $r_{3}=0.701 \cdot r_{1}$ radius correspond to the radii of metal atoms or non-metals and are also capable to form stable quasicrystalline structures. 


\section{Quantum dots in quasicrystalline films in a potential pit model with infinitely high walls}

Traditional mathematical formalism of describing quantum points is in solving stationary Schrödinger equation for a particle in a potential field in the approximation of microparticle effective mass [15]

$$
-\frac{\hbar^{2}}{2 m_{e f f}} \nabla^{2} \Psi+U(r) \Psi=E \Psi .
$$

Potential energy in equation (13) is determined by applied kind of interaction potential between particles. The solution of Schrödinger equation (13) is wave functions

$$
\Psi_{n}(r, \theta, \varphi)=R_{l}(r) Y_{n}(\theta, \varphi)
$$

Energy spectrum of a particle for a spherical quantum point is determined by the following formula [16]

$$
\Delta E_{n, l}=2 \xi_{n, l} \cdot \frac{\hbar^{2}}{m_{r}^{*} D^{2}}
$$

where $\xi_{n, l}-n$ root of spherical Bessel function of half-integer argument $(1+1 / 2)$, with $\xi_{n, 0}=\pi \cdot n(n=1,2,3, \ldots), D$ - spherical diametre.

A number $l=0,1,2, \ldots$ is an orbital quantum number, with a pair of numbers $(n, l)$ entirely defining electromagnetic spectrum of a quantum point. Symbols used for the atomic spectrum indicate its levels. So spherical quantum dots are called artificial atoms.

The ratio for calculation of particle energy spectrum in cylindrical quantum point is described in [17]

$$
\Delta E_{n, l}=\frac{\hbar^{2}}{2 m_{0}}\left(\frac{\pi^{2} n^{2}}{4 h^{2}}+\frac{\xi_{n, l}^{2}}{\rho_{0}^{2}}\right),
$$

where $\rho, h$ is the base radius and half of cylinder height.

A quantum dot having the form of a flattened rotation body (ellipsoid, tablet) is described in [18]. The shape of CD surface is given by the rotation ellipsoid by introducing ellipsoidal curvilinear coordinates. And the wave function (as for the case of a cylindrical quantum point) is represented by the following equation:

$$
\Psi_{n}(r, \theta, \varphi)=\exp (i m \varphi) \cdot \xi_{m}(p R) \cos (k z),
$$

where $\xi_{n, l}$ is Bessel function, $m=0,1,2,3, \ldots$.

The authors say that the boundary conditions of $r$ variable have the same equation for the energy of a particle as in the case of a quantum hole.

Eigenvalues of the particle energy located at the $n$-energy level of a deep potential hole are determined by the known formula [15-17]

$$
E_{n}=\frac{\pi^{2} \hbar^{2}}{2 m l^{2}} \cdot n^{2}, \quad(n=1,2,3, \ldots),
$$


where $l$ - potential hole width, $m$ - particle mass, $\hbar$ - Planck's constant.

Energy level difference

$$
\Delta E_{n+1, n}=\frac{\pi^{2} \hbar^{2}}{2 m l^{2}} \cdot(2 n+1), \quad(n=1,2,3, \ldots) .
$$

$(2 n+1)=F_{i}$ is approximately done with small $n$ values, где $F_{i}$ - Fibonacci numbers. So, we can write the following on basis of (18) and (19) formulas

$$
\omega_{n}=\frac{1}{\hbar} \Delta E_{n+1, n}=\frac{\pi^{2} \hbar}{2 m l^{2}} \cdot(\Phi-1) F_{n},(n=1,2,3, \ldots) .
$$

On basis of (19) formula for wave vector where $F_{n}=1$

$$
\left(\omega_{n} / c\right)=\frac{1}{\hbar \cdot c} \Delta E_{n+1, n}=\frac{\pi^{2} \hbar}{2 m c l^{2}} \cdot(\Phi-1), \mathrm{cm}^{-1} .
$$

In formula (21), the mass of a particle is usually the mass of an electron, then for a rectangular potential hole of $l=0.67 \AA$ width we can get the following based on formula (21)

$$
\left(\omega_{n} / c\right)=\frac{1}{\hbar \cdot c} \Delta E_{n+1, n}=3.6 \cdot 10^{6} \mathrm{~cm}^{-1} .
$$

In this case, the radiation from the quantum point is in ultraviolet frequency area.

Protons in the structure of quantum dots appear because of proton-exchange processes, or because of proton-ion modification of crystals, glasses or quasicrystals used to obtain homogeneous near-surface layers by conducting ion-exchange reactions to a given depth.

In the case of proton capture by a quantum dot, like formula (22), we obtain

$$
\left(\omega_{p n} / c\right)=\frac{1}{\hbar \cdot c} \Delta E_{n+1, n}=\frac{\pi^{2} \hbar}{2 m_{p} c l^{2}} \cdot(\Phi-1)=1750 \mathrm{~cm}^{-1} .
$$

Such a frequency value corresponds to radiation in infrared spectrum zone.

Radiation and absorption of infrared radiation by quantum dots is confirmed experimentally and theoretically [19].

Thus, by selecting metals of a certain grade with atoms radius $r_{1}$ of an irradiating surface and a composition of an irradiating proton-ion stream, it is possible to achieve the formation of quasicrystalline film on the metal surface, which has increased elastic and special optical properties.

It is possible to describe the main patterns of proton-ion implantation processes in proposed model. Proton implantation can be carried out on a cyclotron (for example, on a cyclotron U-120 FTI Tomsk PU). Proton component in pulses with a duration of $\sim 2$ ns was more than $40 \%$. Current and beam energy are $100 \mathrm{~mA} / \mathrm{cm}^{2}$ and $120 \mathrm{keV}$, respectively. Occurrence of damages grid with the period of 10-30 microns caused by an exit to cleavage surface under an exposition of $\mathrm{LiNbO}_{3}$ crystals of an X-cut by protons direct stream [2729]. 


\section{Results and discussions}

It has been shown that in the structure of quasicrystalline films it is possible to form quantum dots, which are potential pits of various shapes with quantized motion of an electron or proton. In the case of an electron in a potential hole, such a hole becomes a source of electromagnetic radiation in ultraviolet field of the spectrum. In the case of a proton in a potential hole, the radiation of a quantum point is in infrared field of the spectrum.

The proposed approach of forming quantum dots in quasicrystalline films allows us to hypothesize the formation of one of the components of infrared radiation by the moon surface and other planets that do not have powerful atmosphere.

The Moon surface is constantly exposed to solar wind, which is a stream of ionized particles. Due to the processes taking place inside the star, the solar wind can have different intensity and speed. The solar wind can be calm and indignant. Calm solar wind flows can be divided into two types: slow and fast. Slow ones have a speed of about $300-500 \mathrm{~km} / \mathrm{s}$. Fast flows have a speed about $500-800 \mathrm{~km} / \mathrm{s}$.

Samples of The Moon soil delivered to The Earth by Soviet and American spacecraft (for example, Luna-20, Fig. 3) made it possible to state chemical composition of The Moon's surface rocks. Information about chemical elements in the Moon's surface rocks (element's percentage in the rocks of the Moon surface is indicated in parentheses), as well as the radius of the first coordination sphere of these elements [20] is in the first column of Table 3.

Table 3. Formation of moon surface quasicrystalline systems [30, 31].

\begin{tabular}{|c|c|}
\hline $\begin{array}{l}\text { The Moon's soil element } \\
\text { (Luna-20) [28] }\end{array}$ & Quasicrystalline system, $r_{1}, \AA[20,21-26]$ \\
\hline $\begin{array}{c}\text { Silicon (Si), } 23 \% \\
r_{1}=1.32 \AA \\
{[20]}\end{array}$ & $\begin{array}{l}r_{2}=0.92 \AA \text { Sulphur. Silicon sulfide is formed. It is a binary } \\
\text { inorganic compound of silicon and sulphur with } \mathrm{SiS}_{2} \text { formula, white } \\
\text { or gray crystals, easily hydrolyzed by water, reacts with oxygen }\end{array}$ \\
\hline $\begin{array}{c}\text { Iron }(\mathrm{Fe}), 13 \% \\
r_{1}=1.56 \AA \\
{[20]}\end{array}$ & $\begin{array}{l}r_{2}=1.09 \AA \text { Phosphorus. Iron-phosphorus alloys are formed, } \\
\text { obtained, and used to restore and increase wear resistance of } \\
\text { machine parts. } \\
\text { Classical methods of producing alloys of iron with phosphorus } \\
\text { (ferrophosphorus) are described in }[18,19]\end{array}$ \\
\hline $\begin{array}{c}\text { Calcium (Ca), } 10 \% \\
r_{1}=1.94 \AA \\
{[20]}\end{array}$ & $\begin{array}{l}r_{2}=1.35 \AA \text { Gallium. CaGa compound is formed, has an } \\
\text { orthorhombic lattice with } a=0.4382 \mathrm{~nm}, \quad b=1.1935 \mathrm{~nm} \text {, } \\
c=0.4196 \mathrm{~nm} \text {. Low temperature modification } \mathrm{CaGa}_{2} \text { has hexagonal } \\
\text { latitude with } a=0.4461 \mathrm{~nm}, c=0.7359 \mathrm{~nm} \text {. CaGa4 has tetragonal } \\
\text { lattice with } a=0.4365 \mathrm{~nm}, c=1.065 \mathrm{~nm} \text {. Ca } 28 \mathrm{Ga}_{11} \text { has a complex } \\
\text { structure consisting of three types of polyhedrons with } \mathrm{Ca} \text { atoms } \\
\text { around Ga atoms }\end{array}$ \\
\hline $\begin{array}{c}\text { Aluminium (Al), } 12.5 \% \\
r_{1}=1.18 \AA \\
{[20]}\end{array}$ & $\begin{array}{l}r_{2}=0.83 \AA \text { Chlorine. Aluminum chloride is formed - colorless } \\
\text { crystals that smoke due to hydrolysis in wet air. At normal pressure } \\
\text { it sublimates at } 183^{\circ} \mathrm{C} \text { (under pressure melts at } 192,6^{\circ} \mathrm{C} \text { ) [14] }\end{array}$ \\
\hline $\begin{array}{c}\text { Magnesium (Mg), } 5.7 \% \\
r_{1}=1.45 \AA \\
{[20]}\end{array}$ & $\begin{array}{l}r_{2}=1.01 \AA \text { Phosphorus. Magnesium phosphide is formed }- \text { a } \\
\text { binary inorganic compound of magnesium and phosphorus with } \\
\mathrm{Mg}_{3} \mathrm{P}_{2} \text {. These are bright yellow cubic crystals }\end{array}$ \\
\hline $\begin{array}{c}\text { Fluorine (F), } 5.1 \% \\
r_{1}=0.42 \AA \\
{[20]}\end{array}$ & $\begin{array}{l}r_{2}=0.29 \AA \text { Helium is formed. Researchers conducted a large-scale } \\
\text { search for possible stable compounds of helium with various } \\
\text { elements (H, O, F, Na, K, Mg, Li, Rb, Cs и т.д.) by using USPEX } \\
\text { code }\end{array}$ \\
\hline
\end{tabular}

Chemical composition of the Mercury surface rocks is almost the same as the composition of the Moon soil. However, the surface of the Mercury is exposed to more 
vigorous proton-ion solar flows. The process of implanting solar flux ions into the Mercury structure surface rocks is accompanied by the formation of quasicrystalline films on the surface of rocks and quantum points in the structure of quasicrystalline films emitting electromagnetic waves in IR range.

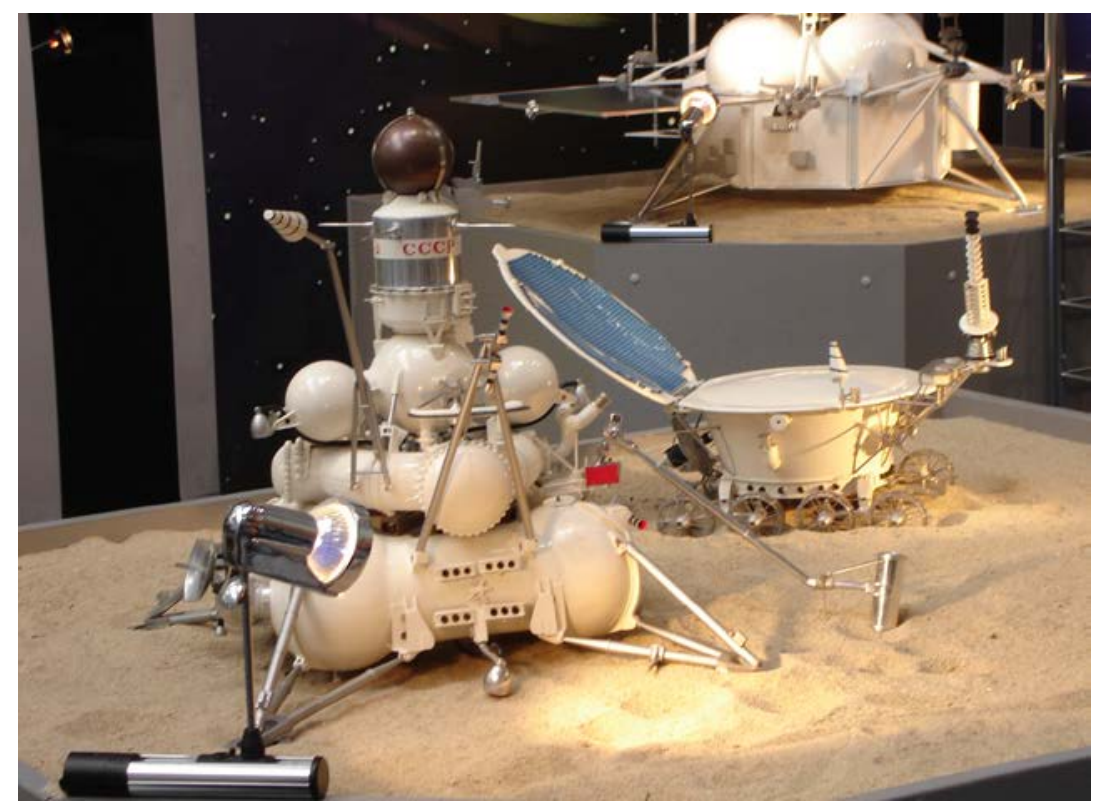

Fig. 3. Exposition of Soviet «moon rockets» models at the exhibition in Paris in 2007. In the foreground - AMS «Luna-20». It includes: a descent vehicle that delivered samples of the Moon soil to the Earth [28].

The Mercury surface acts as an energy converter of solar proton-ion fluxes into infrared radiation by means of proton-ion implantation [31].

\section{Conclusions}

When irradiating the crystal surface with proton-ion flows at a certain ratio of atoms diameters of the irradiated metal and the ions of the irradiating flow, a quasicrystalline film with a dense package of atoms can be formed on crystal surface. Nanostructuring of materials is an entire family of physicochemical processes associated with proton transfer and their localization in crystal lattice and these include ion exchange, diffusion, and ion implantation. Ion and proton exchange can be considered a universal method of surface modification technology [24-26].

The paper considers the case of proton implantation into the structure of cluster systems formed on the surface of crystals. In this case, a quantum dot is formed in the structure of the cluster as a potential well with quantized motion of a proton inside, and the emission of the quantum dot lies in the IR region of the electromagnetic spectrum.

In this proposed work, the main results and mathematical formulas are obtained in the approximation of solid spheres, which is currently one of the exact theoretical methods for studying physical properties of condensed media. A classic example is Carnahan-Starling solid sphere theory for compiling state equation [32]. Prediction of Bernal fluid's structure [33] or the structure of Berry-Smirnov cluster systems [34]. The theory of solid spheres allows further generalization for soft or rough spheres. 


\section{References}

[1] D. Shechtman, I. Blech, D. Gratias, J.W.Cahn, Metallic phase with long-range orientational order and no translational symmetry, Phys. Rev. Lett., v. 53(20), pp. 1951-1953 (1984)

[2] Yu.Kh. Vekilov, M.A. Chernikov, Quasicrystals, Physics-Uspekhi, v. 53:6, pp. 537560 (2010)

[3] L. Bendersky, Quasicrystal with one-dimensional translational symmetry and a tenfold rotation axis, Phys. Rev. Lett., v. 54, p. 2422 (1985)

[4] D. Levine, P.J. Steinhardt, Quasicrystals: a new class of ordered structures, Phys. Rev. Lett., v. 53(26), pp. 2477-2480 (1984)

[5] P.A. Kalugin, A.Yu. Kitaev, L.S. Levitov, $\mathrm{Al}_{0.86} \mathrm{Mn}_{0.14}$ six-dimensional crystal, Letters in JETP, v. 41, p. 119 (1985)

[6] E. Abe, Y. Yan, S.J.R. Pennycook, Quasicrystals as cluster aggregates, Nat. Mater., v. 3(11), pp. 759-767 (2004), doi: 10.1038/nmat124

[7] E. Abe, A. Tsai, Decagonal structure of $\mathrm{Al}_{7} 2 \mathrm{Ni}_{20} \mathrm{Co}_{8}$ studied by atomic-resolution electron microscopy, J. of Non-Crystalline Solids, v. 334-335, pp. 198-201 (2004)

[8] A.E. Madison, Symmetry of quasicrystals, Solid State Physics, v. 55(4), pp. 784-796 (2013)

[9] J.E.S. Socolar, P.J. Steinhardt, Quasicrystals I. Definition and structure, Phys. Rev., v. B34, pp. 596-616 (1986)

[10] Sah Yuvaraj, G.S. Ranganath, Optical diffraction in some Fibonacci structures, Optics Communications, v. 114, pp. 18-24 (1995)

[11] G.A. Melnikov, N.M. Ignatenko, V.G. Melnikov, E.N. Cherkasov, O.A. Manzhos, Structure of Small Clusters and IR Spectrum Condensed Matters, Journal of Nano- and Electronic Physics, v. 7(4), 04087(3pp) (2015)

[12] G. Melnikov, N. Ignatenko, P. Krasnych, V. Melnikov, E. Cherkasov, Formation of cluster systems in condensed matter and IR spectra of liquids, IOP Conference Series: Materials Science and Engineering, v. 110, pp. 012064 (2016)

[13] G.A. Melnikov Clusters Fidonacci in the structure of condensed medium, University News. Physics, v. 9/2, pp. 207-210 (2018)

[14] G.A. Melnikov, S.G. Emelyanov, N.M. Ignatenko, V.G. Melnikov, O.A. Manzhos, University News. Physics, v. 9/2, pp. 203-206 (2018)

[15] Z. Flygge, Problems in quantum mechanics. T.1 (Mir, Moscow, 1974)

[16] N.V. Tkach, Yu.A. Seti, Evolution of quasi-stationary states of an electron in an open spherical quantum dot, Phys., v. 51, issue 5, pp. 979-985 (2009)

[17] T.V. Katanjan, Electronic states and optical properties of a cylindrical quantum dot with a Morse confining potential, Avt. ref. diss. Cand. physical-mat. sciences (Yerevan, 2016)

[18] G.G. Zagrya, O.V. Konstantinov, A.V. Matveentsev, The structure of energy quantum levels in a quantum dot in the form of an oblate body of revolution, Physics and technology of semiconductors, v. 37, issue 3, pp. 234-238 (2003)

[19] A.N. Sofronov, R.M. Balagula, D.A. Firsov, L.E. Vorobiev, A.A. Tonkikh, A.A. Sarkisyan, D.B. Airapetyan, L.S. Petrosyan, E.M. Kazaryan, Absorption of farinfrared radiation by Ge/Si quantum dots, Physics and technology of semiconductors, v. 52, issue 1, pp. 63-67 (2018) 
[20] A.R. Regel, V.M. Glazov, Physical properties of electronic melts (Mir, Moscow, 1980)

[21] N.E. Kalinina, O.A. Kavets, A.K. Fodichuk, Strontium Microalloying of Linear Slavs Used in Rocket and Space Technology, Construction Materials, v. 2/4, pp. 147-149 (2014)

[22] S. Neeleshwar, C.L. Chen, C.B. Teai, Y.Y. Chen, Size-dependent of CdSe quantum dots, Phys. Rev. B., v. 71, pp. 201307(R) (2005)

[23]Z. Deng, K. Zhar, B. Gu at al, New Diluted ftrrjmagnetic semiconductor Li(Zn,Mn)P, with decupled charge and spin djpung, Phys. Rev. B., pp. 81203 (2013)

[24] Yu.E. Lozovik, S.Yu. Volkov, Control of electronic correlations in a spherical quantum dot, FTT, v. 45(2), pp. 345 (2005)

[25] N.In. Korolev, S.E. Starodubtsev, E.N. Bormontov, A.F. Klinsky, Features of the electronic spectrum of an open spherical quantum dot with a delta potential, Condensed Matter and Interphase Boundaries, v. 13(1), pp. 67-71 (2011)

[26] E.A. Talalaev, V.A. Emelyanov, Yu.V. Gorokhov, Lateral pressing of composite bar Al, http://fb.ru/article/334802/silumin---eto-silumin-opisanie-himicheskie-svoystva-iprimenenie

[27] A.N. Sergeev, A.N. Annenkov, S.N. Sutulin, Yu.V. Borodin, Proton modification of oxides and oxide films. Reviews on electronic technology. Series 6. Materials. Issue 1 (1486) (Central Research Institute «Electronics», Moscow, 1989)

[28] A.N. Sergeev, L.A. Osadchev, M.N. Frolova, S.N. Sutulin, Yu.V. Borodin, Homogeneous and heterogeneous levels of oxide systems / // Reviews on electronic technology. Series 6. Materials. Issue 2 (1430) (Central Research Institute «Electronics», Moscow, 1989)

[29] V.E. Gasumyants, S.N. Lykov, D.A. Pshenay-Severin, S.A. Rykov, D.A. Firsov, Dimensional quantization. Part 1 Energy Spectrum nanostructures: textbook. allowance, under the editorship of S.N. Lykov (Publishing house of the Polytechnic University, SPb, 2009)

[30] Soil from the mainland of the moon, Ed. V.L. Barsukov, Yu.A. Surkov (Nauka, Moscow, 1979)

[31] K. Sagan, D. Morrison, Planet Mercury, Earth and Universe, No. 1, (1970)

[32] N.F. Carnahan, K.E. Starling, Equation of State of Noninteracting Rigid Spheres, J. Chem. Phys., v. 51, pp. 635 (1969)

[33] J.D. Bernal, The structure of simple liquids, Proc. Roy. Soc. Lond., v. A280, pp. 299322 (1964)

[34] R.S. Berry, B.M. Smirnov, Phase transitions in clusters of various types, Phys., v. 179(2), pp. 147-177 (2009) 